\title{
Digital mobility services: A population perspective
}

\author{
Joy Goodman-Deane \\ University of Cambridge \\ Cambridge CB2 1PZ \\ UK \\ jag76@cam.ac.uk
}

Nina Nesterova

Breda University of Applied Sciences 4817 JS Breda, The Netherlands

Nesterova.N@buas.nI

\author{
Jakob Kluge \\ Institut für Zukunftsstudien \\ und Technologiebewertung \\ 14129 Berlin, Germany \\ j.kluge@izt.de
}

\author{
Elisabet Roca Bosch \\ Universitat Politènica de Catalunya \\ c/Jordi Girona, 1-3 \\ 08034 Barcelona, Spain \\ elisabet.roca@upc.edu
}

\begin{abstract}
Digital mobility services have great potential to increase passengers' transportation options, improve their experiences and reduce exclusion. For example, they can facilitate access to information and support, and join transport modes together more seamlessly. However, these advantages will only be available to those who can access and use these services effectively. To facilitate the development of usable and inclusive services, information on the range of potential users' digital interface capabilities, attitudes and current use of digital services is needed. A population-representative survey examining these issues was carried out with 1010 participants in Germany in 2020. As well as self-report questions, it examined basic digital interface competence using simplified paper prototyping. The results are examined in terms of the characteristics of groups that are particularly vulnerable to either digital or transport exclusion. Older people (aged $65+$ ), people with disabilities and people with low levels of education were found to have particularly low levels of digital technology access, use, attitudes and competence. Caution is thus required when rolling out digital mobility services. Non-digital alternatives are needed to ensure an inclusive service. When digital interfaces are used, they need to be designed carefully to be usable by and reassuring to digital novices.
\end{abstract}

Inclusive Design. Digital transport. Digital exclusion. Vulnerable to exclusion groups. Older people. Disabilities.

\section{INTRODUCTION}

Digital mobility services have great potential to improve passengers' transportation options and experiences, offering a wide range of mobility innovations to meet changing lifestyles. For example, they can provide better access to information and support, and can help passengers to combine different types of transport modes together for a single journey. Furthermore, they can facilitate essential travel while reducing the need for direct human contact when, and for whom, this is important, for example during a pandemic or for people with communication difficulties. Examples of these services include map applications, route planners, vehicle sharing systems and ticketing and payment facilities.

However, these services will only be useful for people who can access and use them effectively. Despite steady increases in internet use in the EU,
9.5 per cent of the population have never gone online, with large differences between countries and sub-groups (European Commission, 2020). Larger numbers do not own a smartphone (Taylor and Silver, 2019). Furthermore, using the internet or a smartphone does not guarantee the ability to operate complex digital services.

This is a particular issue for digital mobility services because some of the groups that could benefit the most from improved access to transport are also at higher risk of digital exclusion. For example, there is low digital technology use among people with low education, older people and those who retired or inactive (European Commission, 2020). There is a danger that, rather than helping, digital mobility services may exacerbate the existing disadvantages for such groups.

As a result, care needs to be taken in the design of digital mobility services to ensure that they are 
appropriate for and can be used by these groups. To do this, it is important to understand the characteristics and needs of people in general and of vulnerable to exclusion groups in particular, considering aspects such as technology use, digital interface competence, transport needs and current use of digital mobility services.

\subsection{Vulnerable to exclusion groups}

An examination of the literature (e.g. Hoeke et al. 2020, Durand and Zijlstra, 2020) has identified seven groups that are more likely to be affected by digital mobility exclusion:

- Older people: This group has lower levels of technology use and digital interface competence and may also experience mobility issues, capability loss and psychological constraints, such as anxiety, about falling or catching the wrong bus.

- Women: Although many European countries report little gender gap in digital technology use, there are still noticeable gaps in some countries. In addition, women often have lower financial resources and different transport needs and patterns. Inherent biases and differences in attitudes towards technology also play a part.

- People with low levels of education: Education attainment is correlated with a range of digital skills and hence ability to use digital mobility services.

- People with low levels of income: Low income affects access to and ownership of technology devices, as well as car ownership and transport patterns.

- Inhabitants of rural areas: Transport provision and needs, as well as demographic breakdown, differ between rural and urban areas. Rural areas may also lack communication infrastructure (e.g. wireless communications services).

- Migrants: This group may experience barriers to technology and transport use due to language and culture. Some may also have different transportation needs.

- People with disabilities: This group often experiences difficulties with transport use and may require additional information and assistance when travelling. They may also have difficulty with certain interfaces.

Previous research tends to focus on aspects that may cause and exacerbate difficulties for a particular vulnerable group. However, in reality, people belong to multiple groups. Digital division and mobility poverty should be considered as multilayered phenomena (Kuttler and Moraglio 2020; Durand and Zijlstra, 2020).
The study described in this paper adds to this work by providing initial results from a populationrepresentative survey of 1010 adults in Germany. It examines a range of variables of relevance to digital mobility services. This initial analysis in this paper examines the characteristics of each of these vulnerable groups defined above separately but this is merely preparatory to a more in-depth analysis of how the groups interact.

\subsection{The wider project}

The survey described in this paper is part of a larger research project, examining how to foster a sustainable, integrated and user-friendly digital travel eco-system that improves accessibility and social inclusion, along with the travel experience and daily life of all citizens (Dignity project, 2021).

As part of this project, a survey is being conducted in five different European countries (Belgium, Germany, Italy, the Netherlands and Spain). The surveys in some of these countries are still underway, having been delayed due to COVID-19 restrictions. This paper reports on initial results from the German survey, which was the first of these surveys to be completed.

\section{METHOD}

\subsection{Overview of method}

The German survey was conducted by forsa, a German independent market and opinion research institute. Participants completed the questionnaire face-to-face with an interviewer. Each interview took 20 to 30 minutes. Ethical approval was obtained from the University of Cambridge Engineering Department ethics committee.

\subsection{German sample}

The ADM face-to-face sampling system was used in the German survey to obtain a populationrepresentative sample of 1010 adults. The ADM framework is a three-stage stratified random sampling design and is frequently employed in market, media and social research in Germany (Häder, 2016). After the selection of sample locations, private households and target persons within these households were selected at random using a random route procedure. At least four contact attempts were made for each target household or person. No incentives were offered to participants.

The distribution of the sample compared with that in the German population as a whole is shown in Table 1. A weighting variable was calculated to better represent the population, taking region, age and gender into account. The final column of Table 1 and all results presented in this paper use this weighting. 
Table 1: Sample distribution. German population percentages come from the German census, the German Federal Statistical Office, the World Bank, UN DESA and Vuma Touchpoints, obtained through Statista (undated). Figures for education are from Statistisches Bundesamt (undated). Smartphone use in the survey refers to those who used a smartphone at least once a week. Sample percentages are given as a proportion of those who responded to the question.

\begin{tabular}{|l|l|l|l|l|}
\hline Variable & Value & $\begin{array}{l}\% \text { in German } \\
\text { population }\end{array}$ & $\begin{array}{l}\% \text { in unweighted } \\
\text { sample }\end{array}$ & $\begin{array}{l}\text { \% in weighted } \\
\text { sample }\end{array}$ \\
\hline Gender & Male & $49.3 \%$ & $48.4 \%$ & $49.0 \%$ \\
\hline & Female & $50.7 \%$ & $51.6 \%$ & $51.0 \%$ \\
\hline Age & $16-39$ & $33.3 \%$ & $35.9 \%$ & $33.4 \%$ \\
\hline & $40-64$ & $41.2 \%$ & $44.4 \%$ & $41.3 \%$ \\
\hline & $65-74$ & $12.0 \%$ & $12.7 \%$ & $15.1 \%$ \\
\hline Location & $75+$ & $13.5 \%$ & $7.0 \%$ & $10.1 \%$ \\
\hline & Urban & $77.4 \%$ & $71.0 \%$ & $70.7 \%$ \\
\hline use & Rural & $22.6 \%$ & $29.0 \%$ & $29.3 \%$ \\
\hline Education & Use smartphone & $81.7 \%$ & $85.8 \%$ & $81.9 \%$ \\
\hline & Do not use smartphone & $18.3 \%$ & $14.2 \%$ & $18.1 \%$ \\
\hline & No school leaving certificate & $3.6 \%$ & $1.5 \%$ & $2.6 \%$ \\
\hline & $\begin{array}{l}\text { School leaving certificate (secondary general } \\
\text { or intermediate or equivalent) }\end{array}$ & $60.1 \%$ & $1.7 \%$ & $2.0 \%$ \\
\hline & University entrance qualification or higher & $31.9 \%$ & $71.1 \%$ & $62.3 \%$ \\
\hline
\end{tabular}

\subsection{Questionnaire}

The survey questionnaire was adapted from a previous survey conducted in the UK in 2019 (Goodman-Deane et al, 2020). Some questions were omitted or modified based on the experiences in the UK survey and subsequent validation test. A module was added focusing on the use of technology for transport (see Section 2.3.2).

The questionnaire was developed in English and then translated into German and the other survey languages by professional translators. They were translated back into English and checked by the survey creators before adjustments were made and the translations finalised.

The questionnaire covered a range of topics as described below. Most questions were multiplechoice self-report, except for digital interface competence as described in Section 2.3.4.

\subsubsection{Technology access and use}

Participants were asked multiple-choice questions about their access to and frequency of use of the internet, computers, tablets and smartphones. They were then asked whether they had performed various technology activities recently. A first set of questions asked about activities in the last 3 months, and a second set examined activities that are commonly performed less frequently or relate to a deeper knowledge of technology devices, over the last 12 months. A list of activities is given in Section 3.2.
The questions about technology access and use were based on items in the Internet Access Survey 2017 (Office for National Statistics, 2017) to allow for comparison with national UK statistics. The questions were slightly abbreviated from those asked in the UK survey.

\subsubsection{Use of technology for transport}

Participants were asked to rate their confidence in their ability to plan an unfamiliar, local public transport journey using a computer and using a smartphone, on a scale from 1 (Not at all confident) to 10 (Totally confident). This provides an estimate of participants' self-efficacy with digital mobility services in different forms.

Additional self-report questions examined what sources participants used to obtain information about public transport, how often participants used particular digital mobility services, and whether and why participants felt limited in their regular travel within their region.

\subsubsection{Attitudes towards technology}

Overall attitudes towards technology were examined using the ATI (Affinity for Technology Interaction) scale. This examines "whether users tend to actively approach interaction with technical systems or, rather, tend to avoid intensive interaction with new systems" (Franke et al, 2018). The ATI scale comprises nine self-report items with a six-point response scale from "completely disagree" to "completely agree". 
To explore attitudes further, some additional questions were added using the same response scale, examining aspects such as willingness to explore an unfamiliar interface and confidence in using new technology.

\subsubsection{Basic digital interface competence}

This module assessed participants' performance on eight basic digital interface tests using simplified paper prototyping. In each test, the participants were shown a picture of a smartphone interface on a paper showcard. An example is shown in Figure 1. The interfaces were created in English, based on those used in the UK survey, and then adapted for use in different countries with different languages and locations.

Participants were asked to indicate on the showcard what they would do to achieve a particular goal. For example, one of the goals for the interface in Figure 1 was to change the number of adults (Erwachsene) in the accommodation search (Unterkunftssuche). In some cases, achieving a goal might require several actions. Participants were asked to indicate just the first action they would do, by indicating on the showcard. The interviewer coded each response as one of a set of predetermined options. This simplified paper prototyping method was used to keep the length and cost of the interviews down, enabling a larger sample size.

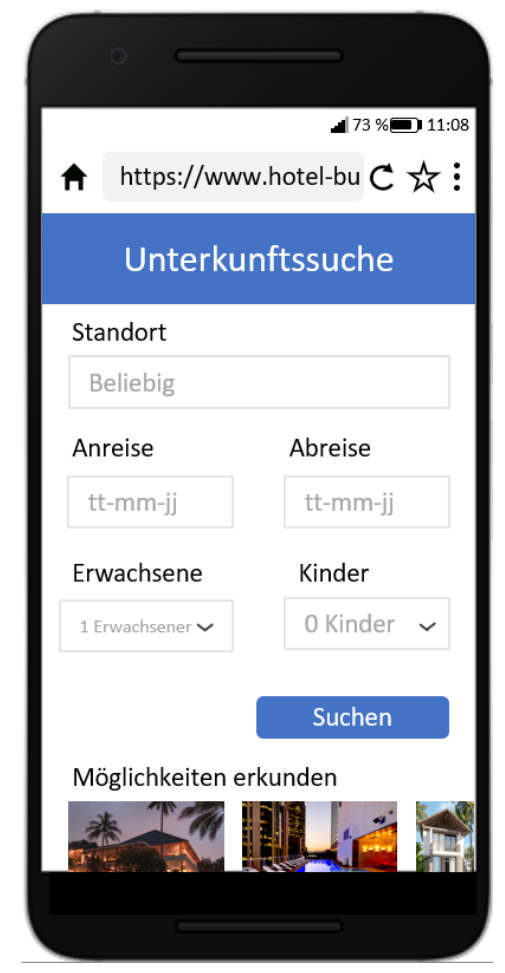

Figure 1: Example of one of the interfaces used in the digital interface competence tests: a mock-up of a website to search for accommodation options.

The interfaces and questions were chosen to cover a range of common, basic digital interface patterns on a smartphone: search, changing settings, creating a new event, opening a menu with more options, going back to a previous screen, activating a drop-down menu, activating an on-screen keyboard and setting favourites. As such, the tests examined a basic level of digital interface competence, rather than the capability to perform complex tasks on a digital device.

\subsubsection{Other modules}

Other modules examined demographics, as well as basic measures of sensory, cognitive and motor capabilities.

\section{RESULTS AND ANALYSIS}

The analysis was conducted in SPSS v27 and the dataset was weighted by region, age and gender to better match the population as a whole. All the results reported in this paper use this weighting.

For brevity and clarity, this paper reports on selected summary results, calculated from the responses to individual questions. These were selected to cover key aspects covered in the survey.

Significance testing was conducted using MannWhitney $U$ tests, comparing each vulnerable group against the rest of the survey sample on each variable of interest. Because the results were weighted, some of the frequency counts were noninteger and had to be rounded to the nearest integer for the analysis. The significance threshold was adjusted to $\mathrm{p}<0.007$ using Bonferroni correction because multiple tests were performed on each variable. Note that, due to the size of the survey sample, differences may be statistically significant but small in magniture. Due to space and the preliminary nature of the analysis, effect size is not analysed in this paper.

\subsection{Definitions of vulnerable to exclusion groups}

The results were examined for each of the groups identified as being particularly vulnerable to digital mobility exclusion (see Section 1.1). These groups, their proportions in the survey sample and their definitions are given below:

- Older people (25.3\% of sample): those aged 65 and over.

- Women (51.0\%): those giving their gender as female.

- Low education (32.2\%): those listing their highest level of education as secondary general school-leaving certificate or below. This roughly corresponds to ISCED levels 0-2 (Eurostat, undated). Those currently attending general school are not included in this group.

- Low income (14.1\%): those with a net monthly household income below a poverty 
line of 1040 euros for a single-person household (Statistisches Bundesamt, 2021). The poverty line for multi-person households was calculated from this based on the OECD-modified household size (OECD, undated).

- Rural inhabitants (28.3\%): those living in a postal code in an area identified as "rural distinct with some densification" or "sparsely populated rural district" according to the official classification from the Federal Office for Building and Regional Planning (BBR). Note that this definition means that some people who are counted as rural may live in small towns.

- Migrants (9.7\%): those who did not acquire German citizenship at birth. This includes both those who acquired it later and those who are not German citizens.

- People with disabilities (15.8\%): those reporting being "very limited" in their daily activities due to issues with their eyesight, hearing, hands, mobility, reach, memory or concentration.

Note that these groups are not independent. In particular, the vast majority (85.5 per cent) of those reporting a disability were aged 55 and over, with 67.8 per cent of them aged 65 and over.

\subsection{General technology access and use}

The survey examined whether participants had access to various kinds of technology. The results for the different groups are shown in Figure 1.

Ownership of "any mobile phone" was generally high, with the lowest level being 89 per cent among people with disabilities. Access to tablet devices was the lowest, with only 42 per cent of the sample as a whole having access to a tablet. This was also very varied, with only 18 per cent of older people having access to one.

Access varies between groups. Older, low education, low income and disability groups had significantly lower rates of access than the rest of the sample on all these technologies (MannWhitney, $p<0.007)$. The other groups did not differ significantly on any of these variables. These lower levels of access were particularly pronounced for digital technologies (i.e. excluding "any mobile phone") among older people and those with disabilities.

Participants were also asked about their technology experience (see Section 2.3.1). A summary variable was created to represent the total number of activities performed recently out of the following 18: e-mail, voice/video internet calls, social media, online news, internet search, finding information about goods/services, buying goods/services, internet banking, booking travel, mapping applications, moving/copying files, moving files between devices, installing software on a computer, installing apps on a smartphone/tablet, changing settings, word-processing, editing photos, video or audio, and writing code.

The results are shown in Figure 2. For presentation purposes, the number of activities were categorised into High (13-18 activities), Medium (6-12) and Low $(0-5)$. They are presented in this order so that a longer bar for the first category represents a higher amount of technology experience. All groups, except rural inhabitants and migrants, reported significantly lower numbers of technology activities than the rest of the sample (Mann-Whitney, $p<0.007)$.

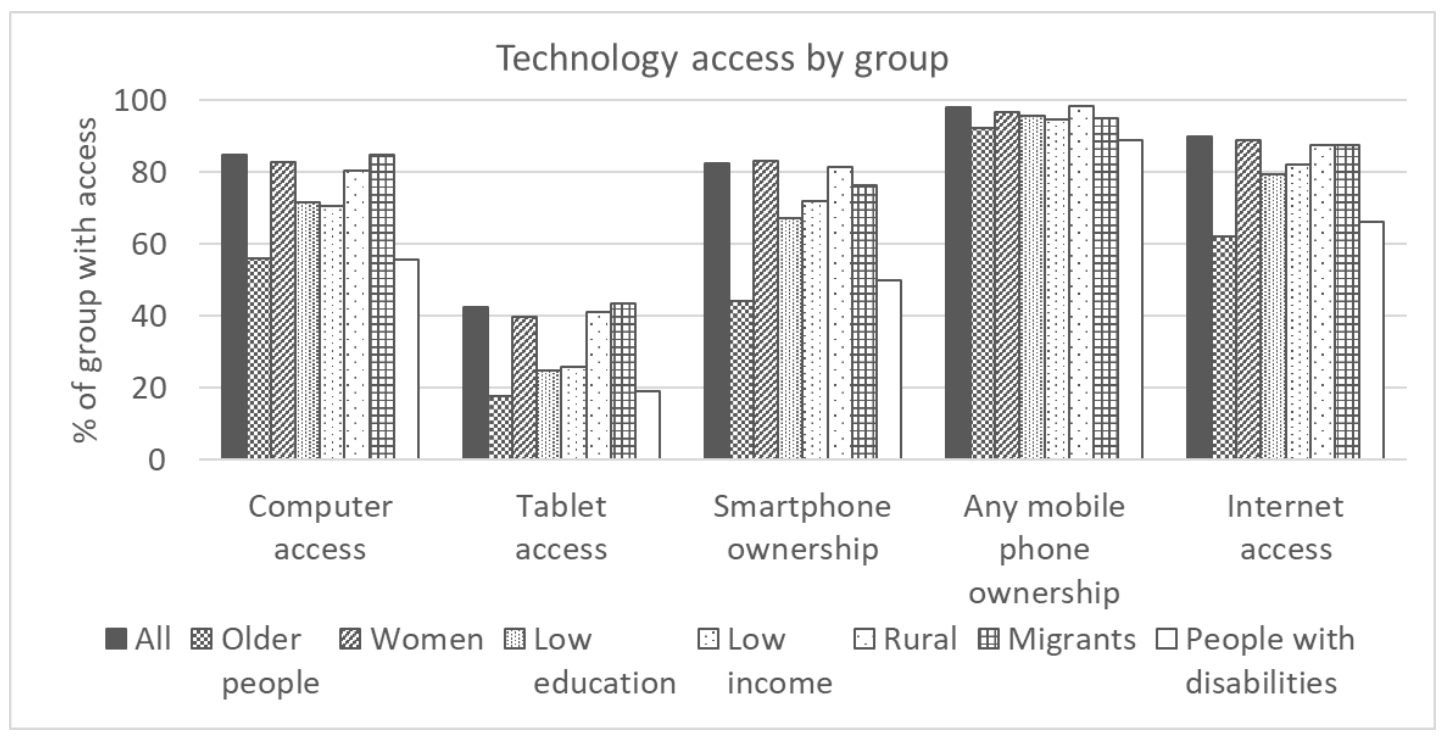

Figure 1: Access to various digital technologies by group. Ownership of smartphones and mobile phones is used rather than general access because these are personal devices. 


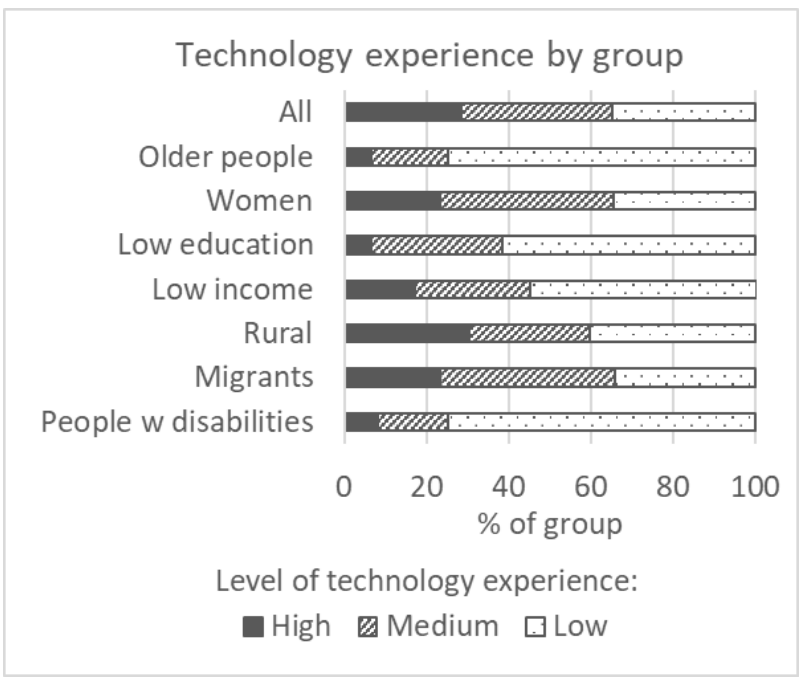

Figure 2: Technology experience by group (based on the number of technology activities conducted recently)

The level of technology experience varied widely between the vulnerable-to-exclusion groups, with particularly low levels amongst older people, people with low education and people with disabilities.

\subsection{Use of technology for transport}

The survey also examined how people obtain information about public transport, e.g. schedules, routes, cancellations and congestion. Participants chose up to three information sources. Figure 3 shows the percentage of each group mentioning any digital information source, such as websites, social media and navigation apps. A lower proportion of older, low education, low income and disabled groups used digital sources than the rest of the sample (Mann-Whitney, $p<0.007$ ).

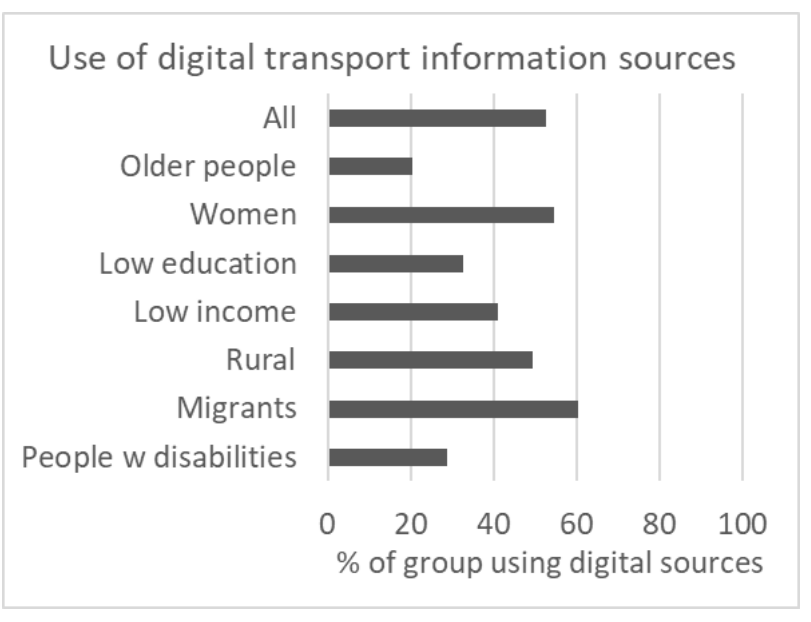

Figure 3: Use of digital information sources about public transport by group

The survey then asked about specific digital mobility services. The figures for the survey as a whole are shown in Figure 4. More detailed response options were used in the survey but are amalgamated into three frequency categories in the graph for visual clarity. 21 per cent of the sample had used any of these digital mobility services in the last 3 months, and 11 per cent at least once a month.

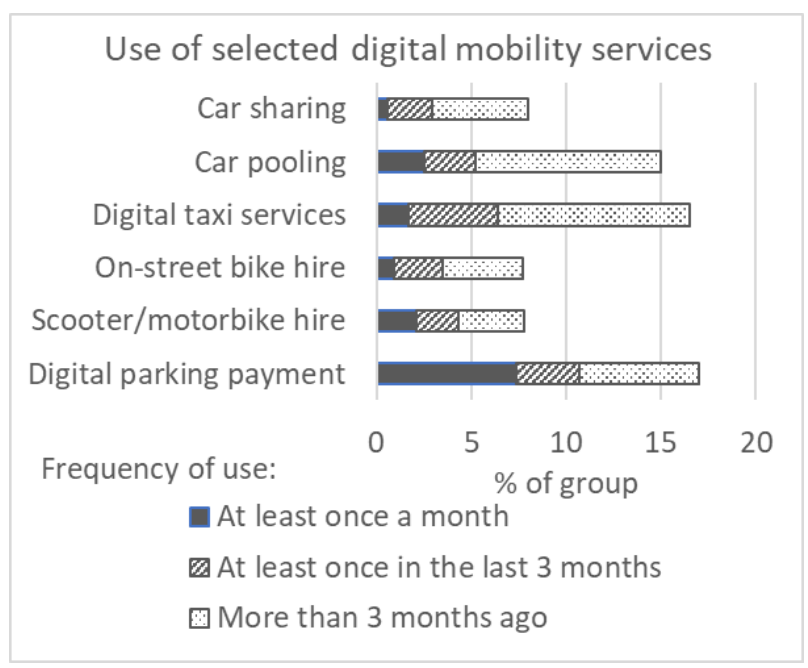

Figure 4: Use of selected digital mobility services in the sample as a whole. There were also a small number of responses of "I don't know" (max $0.6 \%)$, with the remainder replying "Never".

When broken down by group, the numbers for some of the services are extremely small. Thus Figure 5 examines the use of any of the digital mobility services itemised in Figure 4 . There is a big variation between groups. Older people, people with low education and people with disabilities had particularly low usage of these services (MannWhitney, $\mathrm{p}<0.007$ ).

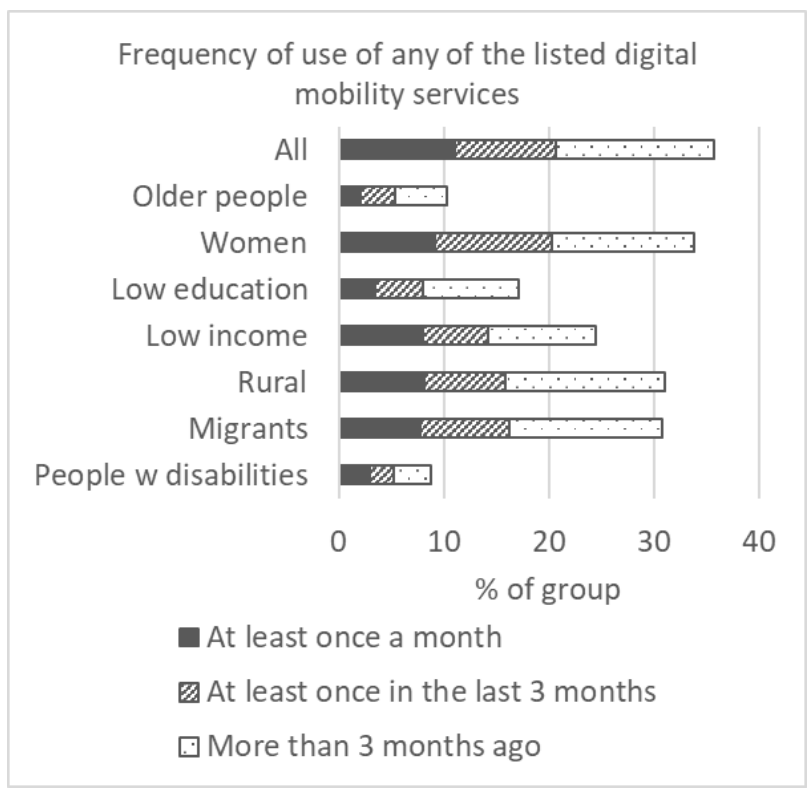

Figure 5: Use of any of the digital mobility services listed in Figure 4 by group.

Participants also rated their confidence in planning a local transport journey using a computer and a smartphone, as shown in Figure 6. For presentation 
purposes, responses were categorised into High (810), Medium (4-7) and Low (1-3).

All groups except rural inhabitants and migrants had significantly lower levels of confidence with both a computer and a smartphone than the rest of the sample (Mann-Whitney, $p<0.007$ ). Older people, people with low education and those with disabilities had particularly low levels of confidence.

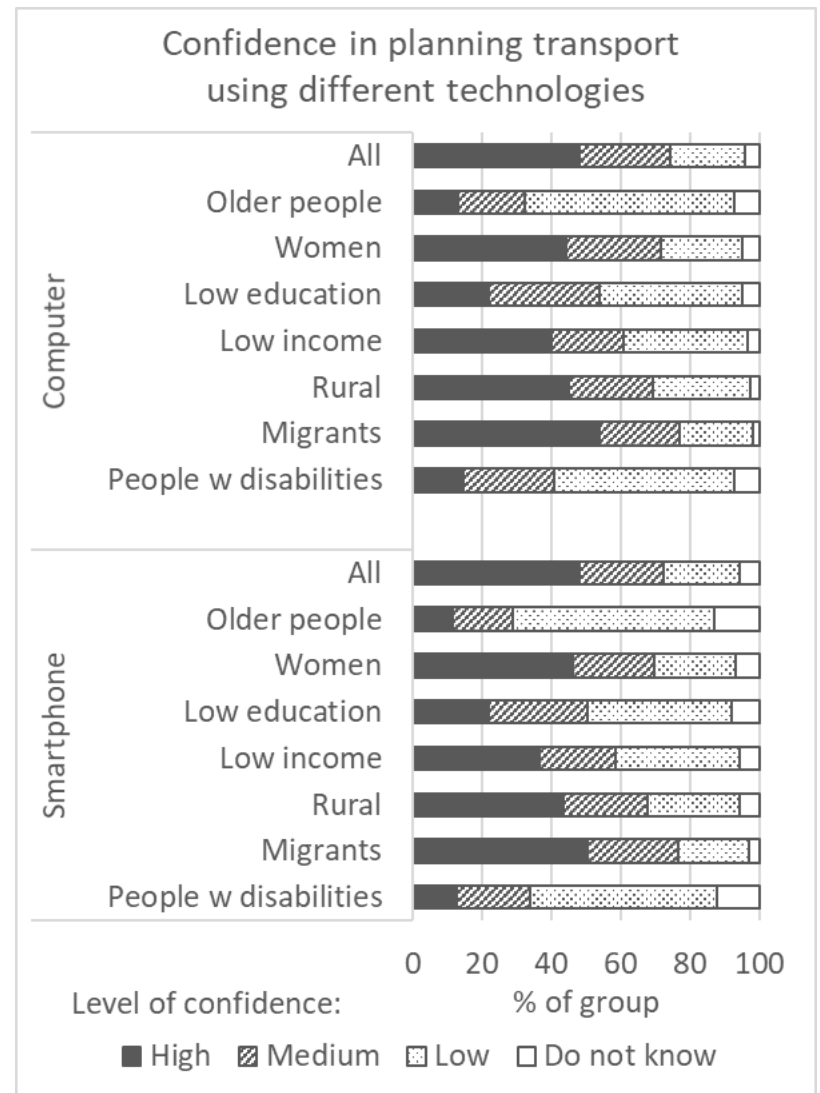

Figure 6: Levels of confidence in planning a local transport journey using a computer and a smartphone

This module also examined whether and why people felt very limited in their regular travel within the region. Figure 7 shows the responses overall and for reasons related to digital skills. The survey examined a range of other reasons for limitations, but this paper focuses on digital aspects.

Higher proportions of the older, female, low education and disabled groups reported feeling very limited because digital skills were needed to plan travel or use the transport (Mann-Whitney, $p<0.007)$. The picture is different when examining limitations in travel for any reason: higher proportions of all groups except women and migrants reported these overall limitations (MannWhitney, $p<0.007)$. The highest levels of limitations were experienced by people with disabilities (of whom 74 per cent felt very limited overall) and older people (65 per cent).

\subsection{Attitudes towards technology}

The ATI (Affinity for Technology Interaction) scale gives each person a score between 1 and 6 . For presentation purposes, these are categorised into High (>4), Medium (3-4) and Low (<3). The results are shown in Figure 8.

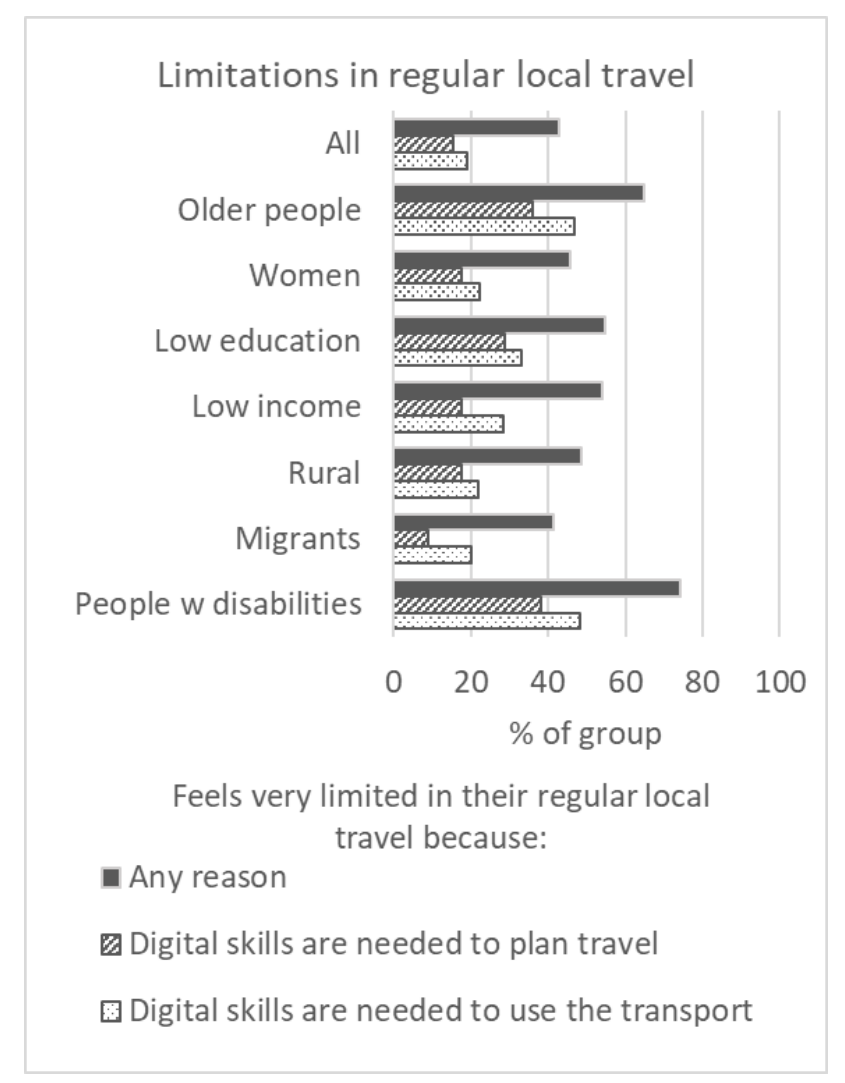

Figure 7: Limitations in regular travel within the region, for any reason and for reasons related to digital skills.

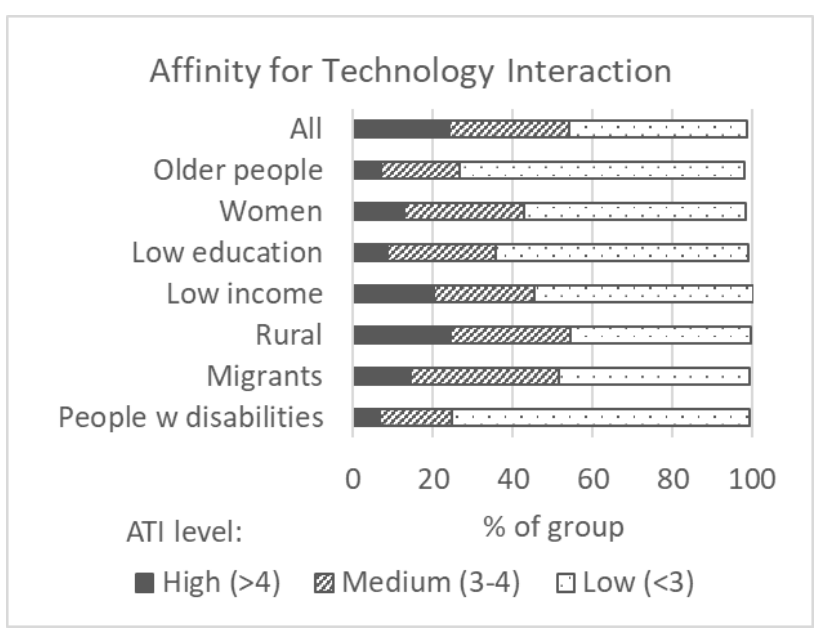

Figure 8: Percentage of each group with Low, Medium and High ATI (Affinity for Technology Interaction) scores

Older people, women and those with low education, and those with disabilities had significantly lower ATI levels than the rest of the sample (Mann-Whitney, $p<0.007)$. Levels were particularly low among older people and those with disabilities. 


\subsection{Basic digital interface competence}

Participants completed eight interface tests as described in Section 2.3.4. Their responses were coded into correct and incorrect, with "I don't know" coded as incorrect. The total number of tests done correctly was calculated. The total was recorded as Missing data if participants declined to do at least half of the tests. The test examined a basic level of digital competence, so the number of tests correct was categorised as described below:

- Low: 4 or fewer tests correct. We estimate that people with these scores are likely to struggle on many modern digital interfaces, particularly on smartphones and tablets.

- Medium: 5 or 6 tests correct. These people are still likely to have some difficulties

- High: 7 or 8 tests correct. a fairly high level of basic digital interface competence. This does not necessarily translate to competence with more complex interfaces and tasks.

The results are shown in Figure 9.

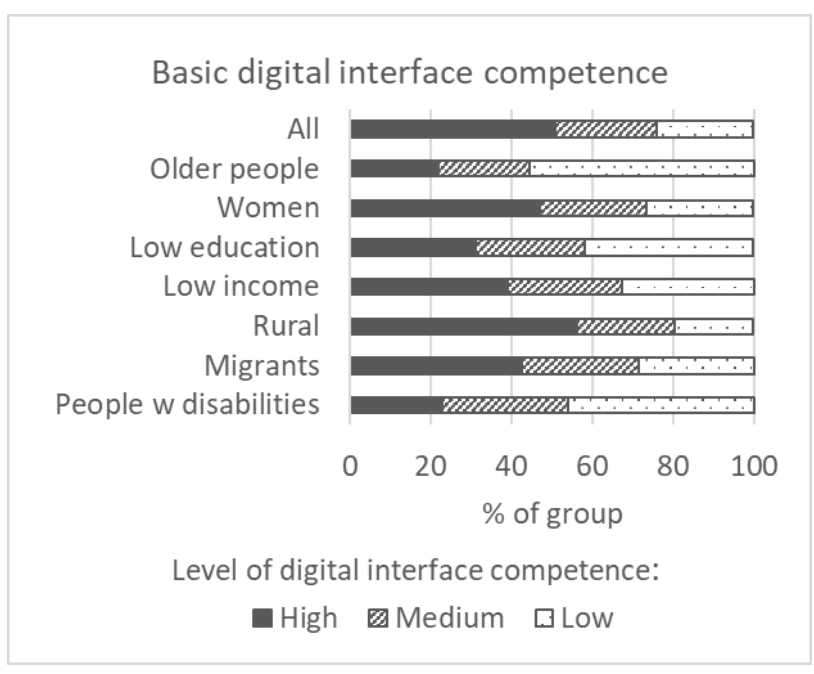

Figure 9: Digital interface competence by group (based on the number of interface tests done correctly)

All groups except rural inhabitants and migrants had significantly lower digital interface competence than the rest of the sample (Mann-Whitney, $\mathrm{p}<0.007$ ), with particularly low levels amongst older people and people with disabilities. Rural inhabitants actually had higher competence levels than the rest of the sample (Mann-Whitney, $p<0.007$ ).

\section{DISCUSSION}

\subsection{Groups with the lowest digital technology use}

Older people reported particularly low levels of digital technology use. This is consistent with previous work which identified negative correlations between age and the use of digital technology in the UK (Goodman-Deane et al, 2020). Similarly, Frid et al (2013) discussed the lower usage of technology among older people in various European countries, and Koch and Frees (2016)'s survey on internet use in Germany found that age was negatively correlated with smartphone usage. The current study extends this work, finding low levels of technology use among older people, both in general and in the context of transport. In addition, this group had low levels of access to a range of technologies, including computers, smartphones, tablets and the internet, as well as more negative attitudes towards technology and lower basic digital interface competence.

In the current analysis, the sample was divided into just two age groups in order to examine a range of vulnerable groups. However, the 65+ age group is large and very varied. Previous work has found that older groups within this range (e.g. 75+) have even lower levels of technology use and competence (e.g. Hargittai et al, 2019). This could be explored further in future work.

Another group with very low levels on all technology variables (including technology access, use, attitudes and competence) were people with disabilities. This may be partly due to the overlap with the older age group, as 68 per cent of those with disabilities were aged 65 and over. However, the group also includes many younger people. Further analysis is needed to explore the intersectionality between these groups, and the differences between younger and older people with disabilities, and between those in each age group with and without disabilities.

This group is heterogeneous in other ways as well as age, including the range of disabilities (sensory, motor and cognitive) and the levels of severity. A range of design adaptations and accessibility features are required to meet these needs.

A third group with low levels on all technology variables is people with low levels of education. This group had slightly higher levels on some of the technology variables than older people or those with disabilities, but still much lower than the rest of the sample. For example, 42 per cent of this group had low levels of digital interface competence as measured by the interface tests, compared to 24 per cent of the sample as a whole.

\subsection{Intermediate groups}

People with low income and women were significantly lower than the rest of the population on some of the variables, but not on others. The size of the difference from the rest of the sample was also smaller than for the groups in Section 4.1. 
People with low income had significantly lower levels of technology access, general technology use and digital interface competence than the rest of the sample. They did not differ in their general attitudes towards technology, but did have lower confidence in planning transport journeys digitally, both using a computer and using a smartphone. A higher proportion reported being very limited in travel due to difficulties during trips, but not prior to travel, because digital skills were needed.

Women reported lower levels of general technology use, attitudes towards technology and competence with technology, but some of the differences were small. They did not differ in their technology access, use of digital transport or overall limitations in travel.

Some previous studies have found gender differences in technology use (OECD, 2018), while others have not. For example, Goodman-Deane (2020b) found no significant gender differences in technology use and competence in the UK. This may be due to differences between countries. Alternatively, the larger sample size $(n=1010)$ in the present study may have enabled detection of smaller differences between groups.

\subsection{Groups with highest technology levels}

At the opposite end of the scale, the survey found that migrants were similar to the sample on all the variables. Rural inhabitants differed only in two variables: a higher proportion of them reported being very limited in travel, but not for reasons related to digital skills. Furthermore, this group had a significantly higher level of digital interface competence than the rest of the sample.

The survey results thus indicate that migrants and rural inhabitants in Germany, considered as groups as a whole, are not at greater risk of digital mobility exclusion on the grounds of general technology access, experience, attitudes and competence. However, they may still have specific needs when it comes to other aspects. For example, the survey did not consider language issues, which are likely to be a particular concern for migrants. The survey did find that rural inhabitants reported greater limitations in transport. Transport needs are different in rural and urban areas, due to the increased distances, logistical issues with transport provision and differences in infrastructure. These should be taken into account when considering digital mobility services in rural areas.

\subsection{Transport services}

The survey found low numbers using the listed digital mobility services: car sharing, car pooling, digital taxi services, on-street bike hire, on-street scooter or motorbike hire and digital parking payment. The usage of these services may have been affected by the Covid-19 pandemic.
Nevertheless, 64 per cent of participants had never used any of these services, with even lower usage in most of the vulnerable groups, especially older people and those with disabilities. This indicates that there is still a long way to go before these services become truly mainstream. Designers and developers should not assume that potential users will know how to access or operate these services. Clear and simple explanations may be required.

Larger numbers used digital sources of information about public transport. 53 per cent of the sample as a whole, and 66 per cent of those who reported using public transport, said they used these information sources. Nevertheless, many do not use them. Digital information sources need to be provided in conjunction with other non-digital means of obtaining important transport information.

The survey also found high levels of mobility poverty, i.e. people who reported feeling limited in their regular travel within their region. 44 per cent of the sample as a whole reported feeling "very limited". Rates within the vulnerable groups varied from 42 per cent for migrants to 74 per cent for people with disabilities. Digitalization of transport products and services is not the only reason for this mobility poverty, but it does play a part, especially for certain groups. 51 per cent of older people, 51 per cent of people with disabilities and 39 per cent of those with low education reported feeling very limited in their travel because digital skills were needed to either plan travel or use transport. This highlights the importance of ensuring inclusivity and usability when rolling out digital mobility services.

\subsection{Design implications and challenges}

It is important for designers and developers to consider carefully who their potential users could be, and what the characteristics of these people are. Particular care is needed if the potential users include older people, people with low education or people with disabilities.

Many older people and people with disabilities do not have internet access (38 and 34 per cent of these groups respectively). Even more (56 and 50 per cent) do not own smartphones. In fact, 18 per cent of population as a whole do not own a smartphone. Thus, while smartphones offer great potential for transport services due to their portability and mobile internet access, they cannot be deployed alone to provide an inclusive service. It is important to offer alternatives. This is highlighted by the numbers of those who are limited in their travel because of requirements to use digital technology, e.g. to access travel information or purchase or present tickets.

One possibility is to offer telephone information and booking lines. The numbers excluded by such services are much smaller, as 98 per cent of the 
sample and 92 per cent of older people own mobile phones. However, even these services do not cover absolutely everyone. In particular, note that visitors to a country may not have mobile signal coverage, or the cost of using a mobile phone may be prohibitive for them.

Another possibility is to offer fixed screens or kiosks displaying information or offering functionality at stops and stations. These overcome the technology access issues, but can still result in exclusion due to the digital interface competence and attitudes of potential users.

As a result, efforts are needed to make any digital interface easier to use, whether on a web browser, smartphone or kiosk. This is particularly important to ensure that people with low digital technology experience and competence are included.

For example, users with low digital interface experience are unlikely to understand the icons, language and conventions of digital interactions. If the target group is likely to include such users, it is important to include text explanations alongside icons. Similarly, these users may be unaware of hidden digital interface conventions and controls. Examples include gestural controls such as 'pinch to zoom'. To prevent exclusion, it is important to provide hints or tips, or offer these interactions in an alternative, more visible format, such as through a zoom button or menu option.

Other issues arise due to attitudes towards technology. Some people are scared or hesitant about using unfamiliar technology, and will not try exploring an unfamiliar interface in case they break something or perhaps buy the wrong ticket. It is thus important to provide clear reassurance and confirmation for actions. Easy and obvious ways to 'undo' an erroneous action also help. As well as increasing the likelihood of successful use, they provide users with reassurance that they can recover from mistakes and give them more confidence to use the system.

\section{CONCLUSIONS AND FURTHER WORK}

This paper has presented results from a survey of 1010 people in Germany in 2020, examining various characteristics related to the use of digital mobility services: technology access, general technology use, attitudes towards technology, basic digital interface competence and the use of technology for transport. It described the characteristics of seven groups that were identified as being particularly vulnerable to either digital or transport exclusion. Older people (aged 65+), people with disabilities and people with low levels of education were found to have particularly low levels of digital technology access, use, attitudes and competence.
The survey also found large numbers of people reporting being very limited in their regular travel because of the need for digital skills to plan travel or use transport. These numbers were particularly high among older people and those with disabilities.

Caution is thus required when rolling out digital mobility services. Non-digital alternatives are needed to ensure an inclusive service. When digital interfaces are used, they need to be designed carefully to be usable by and reassuring to digital novices.

The survey described in this paper is currently underway or completed in four other European countries. An earlier version of the survey was also conducted in the UK. Further work will compare the findings between the countries to get a crossEurope picture.

Further analysis can examine intersectionality, overlaps between groups and differences within a group, e.g. between older and younger people with disabilities, or between smaller age groups within the older population. In addition, the analysis in this paper has only given summaries of key variables. Further analysis could examine more detailed variables, e.g. response to additional questions about attitudes, such as willingness to explore an unfamiliar interface, and performance on particular interface tests. The results could go further to inform the design of inclusive interfaces.

Further work could also compare the results from this survey with qualitative findings from interview and observational studies.

\section{ACKNOWLEDGEMENTS}

This research was done as part of the Dignity project which received funding from the European Union's Horizon 2020 research and innovation programme under grant agreement number 875542 . We would like to thank all the research partners on this project for their input into designing and carrying out the survey design. We are also grateful to Camelia Chivaran from the University of Campania for helping with the descriptive analysis, and to Maribel Ortego from the Universitat Politècnica de Catalunya for advice on the statistical analysis.

\section{REFERENCES}

Dignity project (2021). https://www.dignityproject.eu/ (retrieved May 2021).

Durand, A., Zijlstra, T. (2020) The impact of digitalisation on the access to transport services: a literature review. Netherlands Institute of Transport Policy Analysis.

European Commission (2020) Digital Economy and Society Index (DESI) 2020. https://digital- 
strategy.ec.europa.eu/en/library/digital-economyand-society-index-desi-2020 (retrieved Apr 2021).

Eurostat (undated). International Standard Classification of Education (ISCED) https://ec.europa.eu/eurostat/statisticsexplained/index.php/International_Standard_Cla ssification_of_Education_(ISCED)\#Implementati on_of_ISCED_2011_.28levels_of_education.29 (retrieved Apr 2021).

Franke, T., Attig, C., Wessel, D. (2018) A Personal Resource for Technology Interaction: Development and Validation of the Affinity for Technology Interaction (ATI) Scale. International Journal of Human-Computer Interaction, 35(6), 456-467.

Frid, L., García, A., Laskibar, I., Etxaniz, A., Gonzalez, M.F. (2013). What Technology Can and Cannot Offer an Ageing Population: Current Situation and Future Approach. In Biswas et al (eds) A Multimodal End-2-End Approach to Accessible Computing. Springer-Verlag, London.

Goodman-Deane, J., Bradley, M., Clarkson, P.J. (2020) Digital technology competence and experience in the UK population: who can do what. Ergonomics and Human Factors 2020, Stratford-upon-Avon, UK, Apr 2020. CIEHF.

Häder, S. (2016). Sampling in Practice. GESIS Survey Guidelines. Mannheim, Germany: GESIS - Leibniz.Institute for the Social Sciences. https://www.gesis.org/fileadmin/upload/SDMwiki/ H\%C3\%A4der_Sampling_in_Practice.pdf (retrieved May 2021).

Hargittai, E., Piper, A. M., Morris, M.R. (2019). From internet access to internet skills: digital inequality among older adults. Universal Access in the Information Society, 18, 881-890.

Hoeke, L. Noteborn, C., Goncalves, M.P, Nesterova, N. (2020) Deliverable D1.1 Literature review: Effects of digitalization in mobility in society. Dignity Project. https://www.dignity-project.eu/ wpcontent/uploads/2020/10/200519-D1.1-LiteratureReview-Final.pdf (retrieved Apr 2021).

Koch, W., Frees, B. (2016). Dynamische Entwicklung bei mobiler Internetnutzung sowie Audios und Videos. Ergebnisse der ARD/ZDF-
Onlinestudie. Media Perspektiven, 9, 418-437. https://www.ard-zdfonlinestudie.de/files/2016/0916_Koch_Frees.pdf (retrieved Apr 2021).

Kuttler, T., Moraglio, M. (Eds.) (2020) Re-thinking Mobility Poverty: Understanding Users' Geographies, Backgrounds and Aptitudes (1st ed.). Routledge, London.

OECD (2018) Bridging the digital gender divide: Include, upskill, innovate. Available at: http://www.oecd.org/internet/bridging-the-digitalgender-divide.pdf (retreived May 2021)

OECD (undated) Adjusting household incomes: equivalence scales. https://www.oecd.org/economy/growth/OECDNote-EquivalenceScales.pdf (retrieved Apr 2021).

Office for National Statistics (2017) Internet Access - households and individuals, Great Britain: 2017. https://www.ons.gov.uk/peoplepopulationandcom munity/householdcharacteristics/homeinternetand socialmediausage/bulletins/internetaccesshouseh oldsandindividuals/2017 (retrieved Apr 2021).

Statista (undated) Statistica. https://de.statista.com/ (accessed Apr 2021).

Statistisches Bundesamt (German Federal Statistical Office (undated) Educational attainment. https://www.destatis.de/EN/Themes/SocietyEnvironment/Education-Research-

Culture/Educational-Level/Tables/educationalattainment-population-germany.html (retrieved Apr 2021).

Statistisches Bundesamt (German Federal Statistical Office (2021) Risks of poverty have become further entrenched in Germany. https://www.destatis.de/EN/Press/2021/03/PE21 _113_p001.html (retrieved Apr 2021).

Taylor, K., Silver, L. (2019) Smartphone ownership is growing rapidly around the world, but not always equally. Pew Research Centre, Feb 2019. Available from: https://www.pewresearch.org/global/2019/02/05/ smartphone-ownership-is-growing-rapidlyaround-the-world-but-not-always-equally/ (retrieved May 2021). 